\title{
CONSTRUCTION METHOD OF THE TOPOGRAPHICAL FEATURES MODEL FOR UNDERWATER TERRAIN NAVIGATION
}

\author{
Lihui Wang \\ Le Yu \\ Yanhua Zhu \\ Key Laboratory of micro-inertial instrument and advanced navigation technology, \\ Ministry of education, School of instrument science and engineering, \\ Southeast University Nanjing 210096, \\ China
}

\begin{abstract}
Terrain database is the reference basic for autonomous underwater vehicle (AUV) to implement underwater terrain navigation (UTN) functions, and is the important part of building topographical features model for UTN. To investigate the feasibility and correlation of a variety of terrain parameters as terrain navigation information metrics, this paper described and analyzed the underwater terrain features and topography parameters calculation method. Proposing a comprehensive evaluation method for terrain navigation information, and constructing an underwater navigation information analysis model, which is associated with topographic features. Simulation results show that the underwater terrain features, are associated with UTN information directly or indirectly, also affect the terrain matching capture probability and the positioning accuracy directly.
\end{abstract}

Keywords: Terrain Aided Navigation, terrain features, Terrain Navigation Information Content (TNIC), correlation

\section{INTRODUCTION}

Terrain Aided Navigation (TAN) combined the terrain database with terrain contour matching, which improved the positioning accuracy of inertial navigation of autonomous underwater vehicle (AUV) [1,2]. Terrain Elevation Matching (TEM) is the key technology of TAN. Once system hardware performance and matching area have been identified, the performance of TEM determines the navigation accuracy of TAN. The accuracy and reliability of underwater terrain navigation (UTN) depend on the richness of the terrain information [3-5]. The more abundant of the underwater terrain features, the better the robustness of matching algorithm is, and the more reliable matching results are. Analysis algorithms for different terrain navigation performance, including choosing terrain matching area, have been a focus research of TAN, and are an obstacle for TAN widely used.

One core of terrain matching aided navigation system is the characteristics of the terrain [6,7]. As local statistical characteristics of the terrain, terrain information is a property of terrain itself, which is independent of the specific terrain matching algorithm $[8,9]$. Therefore, this paper established an analysis model of UTN, theoretically analyzed the measures of terrain height standard deviation, Fisher terrain information and terrain elevation entropy, these terrain features which are closely related to the amount of information and terrain navigation, and their impacts on matching performance, and discussed the weighting comprehensive assessment of topographic information. 


\section{A TERRAIN NAVIGATOR ANALYSIS MODEL}

East to the location, the position of the north, east velocity, north velocity are considered as a state quantity of the "position velocity model" of UTN [10]. East acceleration and north acceleration are regarded as the control amount. East velocity, north velocity and water depth $\mathrm{f}$ are regarded as dynamic model measurements.

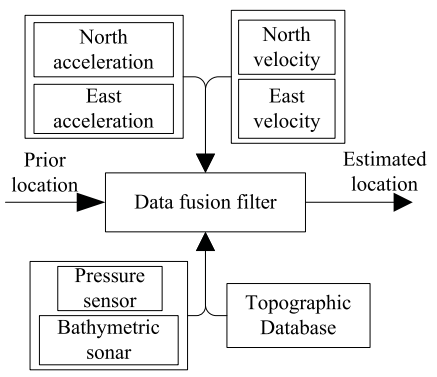

Fig. 1. Schematic diagram of the underwater terrain navigation simulation system

State vector:

$$
\mathbf{x}(t)=\left[\begin{array}{llll}
x(t) & y(t) & v_{x}(t) & v_{y}(t)
\end{array}\right]^{\mathrm{T}}
$$

Control vector:

$$
(t) \quad\left[a_{x}(t) \quad a_{y}(t)\right]
$$

Measurement vector:

$$
\mathbf{z}(t)=\left[\begin{array}{lll}
v_{x}(t) & v_{y}(t) & h_{r}(t)+h(t)
\end{array}\right]^{\mathrm{T}}
$$

System Model:

$$
\begin{aligned}
\dot{\mathbf{x}}(t) & =\mathbf{A x}(t)+\mathbf{B u}(t)+\mathbf{L w}(t) \\
& =\left[\begin{array}{llll}
0 & 0 & 1 & 0 \\
0 & 0 & 0 & 1 \\
0 & 0 & 0 & 0 \\
0 & 0 & 0 & 0
\end{array}\right]\left[\begin{array}{c}
x(t) \\
y(t) \\
v_{x}(t) \\
v_{y}(t)
\end{array}\right]+\left[\begin{array}{ll}
0 & 0 \\
0 & 0 \\
1 & 0 \\
0 & 1
\end{array}\right]\left[\begin{array}{l}
a_{x}(t) \\
a_{y}(t)
\end{array}\right]+\left[\begin{array}{ll}
0 & 0 \\
0 & 0 \\
1 & 0 \\
0 & 1
\end{array}\right]\left[\begin{array}{l}
w_{a_{x}}(t) \\
w_{a_{y}}(t)
\end{array}\right]
\end{aligned}
$$

respectively, both are zero-mean white noise. Discrete state points are estimated by way of the UKF system model. In case that error in the initial position is not so high, UKF is capable of real-time navigation system error correction with high matching accuracy, it has a better prospect of engineering application. Without linearization, we can use the discrete state differential equations directly.

$$
\begin{gathered}
\mathbf{x}_{k}=F\left(\mathbf{x}_{k-1}, \mathbf{u}_{k-1}, \mathbf{w}_{k-1}\right)=\Phi_{k \mid k-1} \mathbf{x}_{k-1}+\mathbf{B}^{*} \mathbf{u}_{k-1}+\mathbf{L}^{*} \mathbf{w}_{k-1} \\
\mathbf{z}_{k}=h\left(\mathbf{x}_{k}\right)+\mathbf{e}(k)=\left[\begin{array}{c}
v_{x}(k) \\
v_{y}(k) \\
h_{d}\left(x_{k}, y_{k}\right)
\end{array}\right]+\left[\begin{array}{c}
e_{v_{x}}(k) \\
e_{v_{y}}(k) \\
e_{h}(k)
\end{array}\right]
\end{gathered}
$$

\section{UNDERWATER TERRAIN NAVIGATION INFORMATION OF EXPRESSION}

Some statistical characteristics of the underwater topography (such as terrain height entropy), directly or indirectly, are related with the amount of information about the terrain navigation $[4,11]$. These features are characteristic of the spatial shape of the ground described with an intuitive meaning [9]. The terrain height standard deviation, Fisher terrain information and terrain elevation entropy, those parameters reflect the size of the terrain informative to some extent.

\section{THE UNDERWATER TOPOGRAPHY DEPTH STANDARD DEVIATION}

The terrain standard deviation reflects the high degree of deviation from the scope of the terrain average terrain elevation, DTED map the terrain matching region into discrete square area $\mathrm{A}$. Set to be investigated terrain elevation sequence $h(\mathrm{i}, \mathrm{j})$ :

$$
\mathbf{z}\left(t_{k}\right)=h\left(\mathbf{x}\left(t_{k}\right)\right)+\mathbf{e}\left(t_{k}\right)=\left[\begin{array}{c}
v_{x}\left(t_{k}\right) \\
v_{y}\left(t_{k}\right) \\
h_{d}\left(x\left(t_{k}\right), y\left(t_{k}\right)\right)
\end{array}\right]+\left[\begin{array}{c}
e_{v_{x}}\left(t_{k}\right) \\
e_{v_{y}}\left(t_{k}\right) \\
e_{h}\left(t_{k}\right)
\end{array}\right]
$$$$
\left\{\begin{array}{l}
\sigma=\sqrt{\frac{1}{m(\mathrm{n}-1)} \sum_{i=1}^{m} \sum_{j=1}^{n}[h(\mathrm{i}, \mathrm{j})-\bar{h}]^{2}} \\
\bar{h}=\frac{1}{m n} \sum_{i=1}^{m} \sum_{j=1}^{n} h(\mathrm{i}, \mathrm{j})
\end{array}\right.
$$

where $\mathrm{w}(\mathrm{t})$ and $\mathrm{e}(\mathrm{t})$ are respectively the process noise and measurement noise, which covariance matrices are $\mathrm{Q}$ and $\mathrm{R}$ is unrelated to viewing direction. $h(i, j)$ indicates the depth reference for reference map. $h$ is the average depth of the local computation window. In most cases, the average elevation 
of the terrain elevation value reflects the topography of the central gathering.

\section{FISHER TERRAIN INFORMATION}

The famous Cramer - Lao (Cramer -Rao) Fisher information inequality can measure topographic information which contains the amount of terrain information [12].

$$
F I C=\sqrt{\frac{1}{N} \sum_{i=1}^{N}\left\|\nabla h\left(x_{i}\right)\right\|^{2}}
$$

In the formula, $x \mathrm{i}$ is flat position that coordinates point in matching region which is uniform distributed. The range of Fisher terrain information content is from 0 to infinity, in fact terrain Fisher quantity can't be infinity, and its upper bound is a big positive number. Fisher information matrix which is common in estimate problem will be introduced.

$$
\begin{gathered}
J=E\left(-\nabla_{X} \nabla_{X}^{T} \log \mathrm{p}(\mathrm{X}, \mathrm{y})\right)= \\
=\frac{1}{\lambda} E\left(\nabla_{X} \mathrm{~h}(\mathrm{X}) \nabla_{X}^{T} \mathrm{~h}(\mathrm{X})-(\mathrm{y}-\mathrm{h}(\mathrm{X})) \nabla_{X} \nabla_{X}^{T} \mathrm{~h}(\mathrm{X})\right)
\end{gathered}
$$

where $h(i, j)$ is a group of sample values which is uniform distributed in the region. Fisher information matrix $J$ defines the sampling mean.

$$
\hat{J}=\left.\left.\sum_{i=1}^{N} \nabla_{X} h(\mathrm{X})\right|_{X=X_{i}} \nabla_{X}^{T} h(\mathrm{X})\right|_{X=X_{i}}
$$

It can be used as a quantity in DTED map, and it has nothing to do with the noise variance $\lambda$ and the area of $A$ (small square area). Obviously

$$
\lim _{n \rightarrow \infty} \frac{1}{\lambda A} \hat{J}=J
$$

where $J$ can be used to measure the amount of information in different terrain region of DTED map. Terrain Fisher quantity FIC is proportional to the sample mean $J$ of Fisher information matrix J, reacted the size of Fisher information matrix J to some extent. So it can link to C-R inequality.

\section{THE TERRAIN ENTROPY}

According to the definition of entropy, entropy indicates the average degree of uncertainty system. Terrain entropy represents uncertainty of terrain height, set a square region $S$ in arbitrary DEM map, corresponding to a terrain entropy $\mathrm{Hf}$ $(i, j)$, which included $n$ elements position sets and $m$ terrain height value sets in the DEM map, it can be defined as

$$
\left\{\begin{array}{l}
P_{i}(\mathrm{i}, \mathrm{j})=\frac{h(\mathrm{i}, \mathrm{j})}{\sum_{i=1}^{m} \sum_{j=1}^{n} h(\mathrm{i}, \mathrm{j})} \\
H_{f}(\mathrm{i}, \mathrm{j})=-\sum_{i=1}^{m} \sum_{j=1}^{n} P_{i}(\mathrm{i}, \mathrm{j}) \log \mathrm{P}_{i}(\mathrm{i}, \mathrm{j})
\end{array}\right.
$$

Local terrain entropy reflects the quantity of the information contained in the terrain, so the local terrain entropy can describe the nature of the terrain [14]. If a local area elevation values change more dramatic, and undulating changes greater, the more unique topography is, the smaller calculated entropy of local terrain is, otherwise, the entropy is greater. Smaller entropy area is suitable for matching area. The range of terrain height entropy vary from 0 to $\lg \mathrm{N}, \mathrm{N}$ represents the calculation of the number of representatives, to the image element within the window of sample values. Terrain height equal to 0 indicates that the entropy calculation window is a flat terrain, that means values of all the pixels is the same height. The calculation is equal to $\lg \mathrm{N}$ represents the window is a rugged terrain terrain, every point is different height values.

\section{THE AMOUNT OF TERRAIN CODING DISTORTION}

From the perspective of information theory, plane position information is the source, and the terrain height value is the signal transmitted in the channel after information source coding $[13,15]$. Physical terrain matching area plays the role of the encoder, which provides the terrain height values by plane location information. Generally, physical terrain matching area is the encoder with a distortion. Usually, because in a certain region with the terrain matching area, it is impossible that terrain height is not the same at all locations, yet there is always one position with a same terrain height value, the terrain matching region is generally not an one to one encoder. The random error coding between encoding input and decoding output is called distortion, the function of distortion amount $\mathrm{d}(\mathrm{xi}, \mathrm{xj})$ represents the distortion of location xi turning into $\mathrm{xj}$ after decoding the encode.

$$
\begin{gathered}
d\left(x_{i}, x_{j}\right)=\left\|x_{i}-x_{j}\right\|= \\
=\sqrt{\left(\mathrm{x}\left(\mathrm{x}_{i}\right)-\mathrm{x}\left(\mathrm{x}_{j}\right)\right)^{2}+\left(\mathrm{y}\left(\mathrm{x}_{i}\right)-y\left(\mathrm{x}_{j}\right)\right)^{2}}
\end{gathered}
$$

The average value $\bar{d}$ of the function of distortion amount $\mathrm{d}(\mathrm{xi}, \mathrm{xj})$ is able to be the distortion amount to express terrain matching region encoding.

$$
\bar{d}=\sum_{i=1}^{N} p\left(\mathrm{x}_{i}\right) \sum_{j=1}^{N} p\left(x_{i}, x_{j}\right) d\left(\mathrm{x}_{i}, \mathrm{x}_{j}\right)
$$

The distortion amount $\bar{d}$ is one of the important indicators to balance the performance of an encoder, ranging from 0 to 
$\mathrm{N} / 2$, and $\mathrm{N}$ being the side of the square calculating window (the number of pixels).if the value of terrain encoding distortion amount equals to 0 , then it represents the area is one with abundant information in the calculating window. By the terrain height value, we can estimate plane location, however, supposing that the amount is $\mathrm{N} / 2$, then it represents the area is a one with little information in the calculating window, which is not suitable for being a terrain matching region.

\section{COMPREHENSIVE EVALUATION METHOD}

The topographic features may be interrelated. In order to find out quantitative relationship between positioning accuracy of matching area and terrain features, assuming there are polynomial relations combining the positioning error mean square with these parameters by regression analysis [10], as following formula:

$$
\begin{gathered}
R M S=\alpha_{0}+\alpha_{1} \sigma_{T}+\alpha_{2} F I C+\alpha_{3} E N T+ \\
+\alpha_{4} D I S+\alpha_{5} \sigma_{T} F I C+\alpha_{6} \sigma_{T} E N T+\alpha_{7} \sigma_{T} D I S \\
-\alpha_{8} F I C * E N T+\alpha_{9} F I C * D I S+\alpha_{10} E N T * D I S- \\
+\alpha_{11} \sigma_{T}^{2}+\alpha_{12} F I C^{2}+\alpha_{13} E N T^{2}+\alpha_{14} D I S^{2}
\end{gathered}
$$

RMS represents positioning error mean square of terrain matching algorithm, $\sigma_{T}$ represents the depth of standard deviation. FIC represents terrain fisher character information, ENT represents terrain height entropy. DIS represents the amount of coding distortion terrain, $\alpha_{0} \sim \alpha_{14}$ represent the coefficients. These terrain features don't represent all of the terrain features, the polynomial is not necessarily universal adaptability, but it can reflect the sensitivity of terrain to some extent.

\section{SIMULATION OF UNDERWATER TERRAIN NAVIGATION INFORMATION}

Through the experimental analysis of the relationship between terrain parameters and navigation errors, terrain parameters used to choose the terrain can be determined. This article is wrote by being measured from the depth of standard deviation, Fisher terrain information, terrain entropy, coding distortion amount of terrain measure and its impact on terrain matching. Figure 2 shows standard deviation of water depth and topography. All measurements were carried out utilizing Monte Carlo simulation. Terrain height standard deviation and the degree of information-rich of terrain feature are corresponding, terrain standard deviation values are large in local area where owning more violent topography and rich feature information, while terrain standard deviation values are small in local area where owning slow topography and deficient feature information. So the local topography standard deviation can be used as a quantitative index to measure the amount of information of the terrain feature. Figure 3 shows Fisher features information. Qualitative say, terrain matching area with bigger FIC contains bigger information of terrain navigator. Compared with small FIC of flat area, FIC of area on undulating terrain is large. Figure 4 shows height entropy of terrain. The terrain is more unique and the information of this area is richer. Flat area has small height entropy while rugged area has large height entropy. Terrain coding distortion of flat area is large and rugged area is small. The smaller the terrain coding distortion is, the more terrain navigation information area contain, the more suitable match for the terrain height. Therefore, when selecting terrain matching area, you should try to choose a small amount of terrain coding distortion.

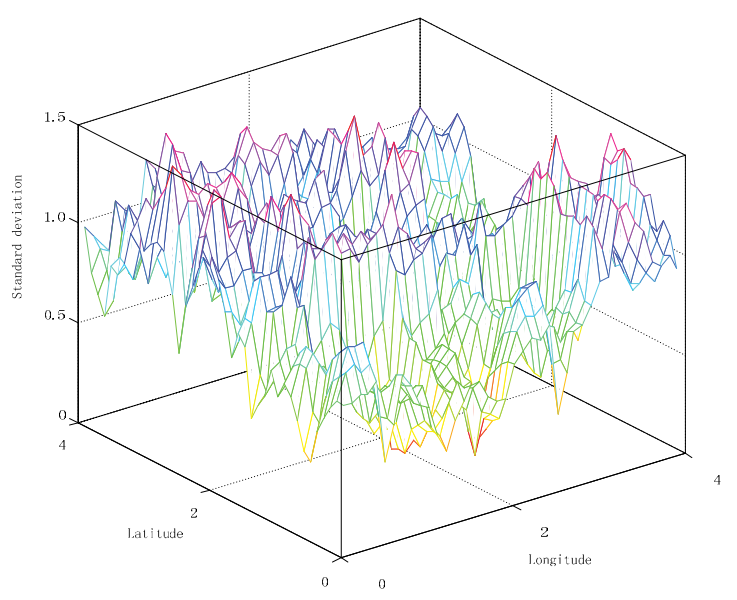

Fig. 2. The standard deviation of water depth and topography

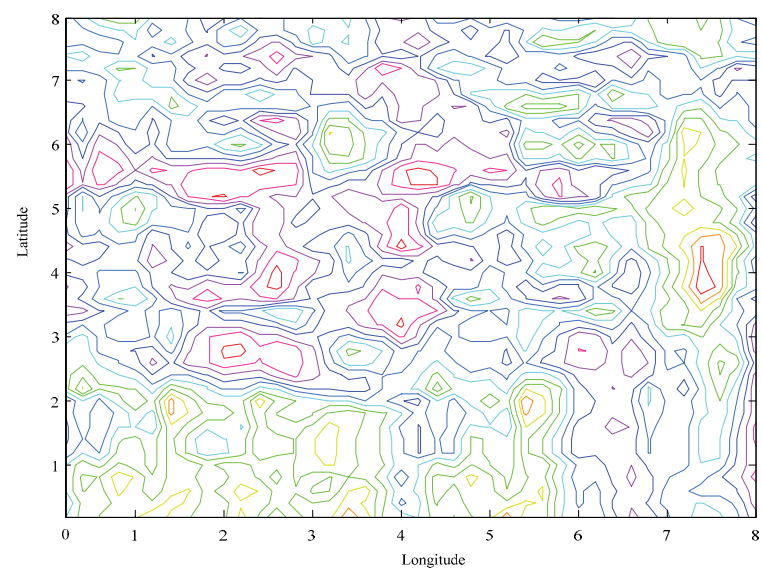

Fig. 3. Fisher terrain features information

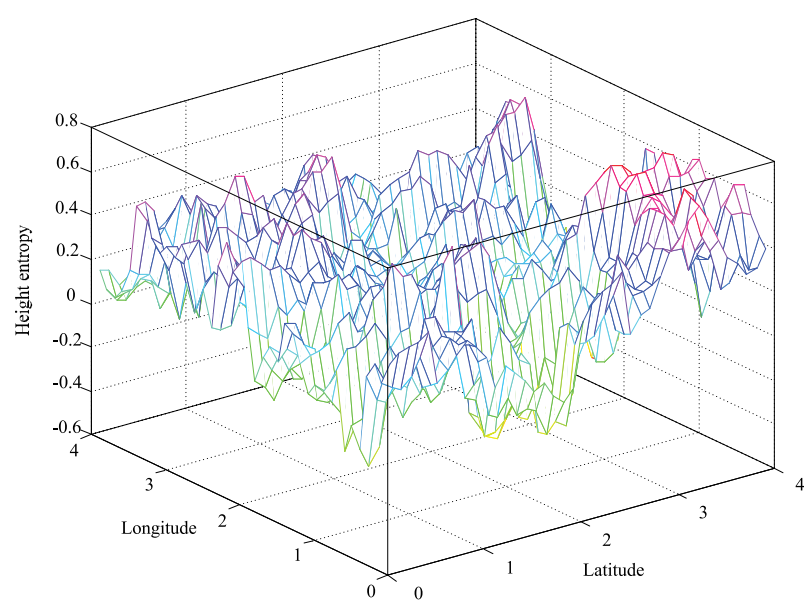

Fig. 4. Height entropy of terrain 


\section{CONCLUSIONS}

Terrain contour matching aided navigation is a hot research direction in recent years. However, besides matching algorithm, matching performance also related to the the amount of information of matching area. By using the UKF nonlinear filtering, we established models of the underwater terrain analysis, obtained that terrain height standard deviation, Fisher terrain information and terrain elevation entropy, then, built quantitative relationships with matching performance, and simulated the models. We presented methods of terrain features correlation analysis, which can be used as a reference standard to select matching area in underwater terrain navigation.

\section{ACKNOWLEDGMENTS}

The project is supported by the following funds: National Natural Science Foundation of China (61203192, 51477028), Fundamental Research Funds for the Central Universities (2242013R30016), Natural Science Foundation of Jiangsu Province (BK2012326, BK20130628), Research Fund of China Ship Industry (13J3.8.4), Foundation of Key Laboratory of Micro-Inertial Instrument and Advanced Navigation Technology, Ministry of Education (201103).

\section{REFERENCES}

1. Anonsen, K.B. and Hagen, O.K., An analysis of real-time terrain aided navigation results from a HUGIN AUV, OCEANS, Vol. 10, pp. 1-9, 2010.

2. Anonsen, K.B. and Hagen, O.K., Terrain aided underwater navigation using pockmarks, OCEANS, pp. 1-6, 2009.

3. Ayanna, M.H., Fuzzy-logic based selection of surface feature observations for small body proximity operations, WAC ‘ 06 . World Automation Congress, pp. 1-6, 2006.

4. Bagnell, J. A., Bradley, D., and Silver, D., et al., Learning for autonomous navigation, Robotics \& Automation Magazine, Vol. 17, no. 2, pp. 74-84, 2010.

5. Carreno, S., Wilson, P., Ridao, P., et al., A survey on terrain based navigation for AUVs, OCEANS,Vol. 10, pp. 1-7, 2010.

6. Deborah K. Meduna, Stephen M. Rock, Robert S. McEwen. Closed-loop terrain relative navigation for AUVs with non-inertial grade navigation sensors, 2010 IEEE/OES Autonomous Underwater Vehicles (AUV),pp. 1-8. 2010.

7. Feng, Q.T., A new method of terrain matching and its environmental adaptability reseach, University of Defense Technology, 2004.

8. Kjetil Bergh Anonsen, Ove Kent Hagen. Recent developments in the HUGIN AUV terrain navigation system, OCEANS, Vol. 9, pp. 1-7, 2011.
9. Nordlund, P.J. and Gustafsson, F., Marginalized particle filter for accurate and reliable terrain-aided navigation, Aerospace and Electronic Systems, Vol. 45, no. 4, pp. $1385-$ 1399, 2009.

10. Marvin, W., Roe, M.E., and Trenchard, M.C.L., Integrating vector overlay information into naval digital map systems. IEEE/AIAA 30th Digital Avionics Systems Conference, pp. 6B5-1 - 6B5-9, 2011.

11. McPhail, S., Stevenson, P., Pebody, M., et al., Challenges of using an AUV to find and map hydrothermal vent sites in deep and rugged terrains, 2010 IEEE International Conference on Autonomous Underwater Vehicles (AUV), pp. 1-8, 2010.

12. Panahandeh, Ghazaleh, Jansson, Magnus. Visionaided inertial navigation using planar terrain features. International Conference on Robot, Vision and Signal Processing, pp. 287-291, 2011.

13. Pullen, J.D. and Allen, J.S., Modeling studies of the coastal circulation off Northern California: shelf response to a major Eel river flood event, Continental Shelf Research, Vol. 20, pp. 2213-2238, 2000.

14. Reynaud, S. and Louis, C., A universal navigability map building approach for improving Terrain-Aided-Navigation accuracy. 2010 IEEE/ION Conference on Position Location and Navigation Symposium (PLANS), pp. 888-896, 2010.

15. Stalder, S., Bleuler, H., and Ura, T., Terrain-based navigation for underwater vehicles using side scan sonar images. OCEANS, pp. 1-3, 2008.

\section{CONTACT WITH THE AUTHORS}

$$
\begin{gathered}
\text { Lihui Wang } \\
\text { Le Yu } \\
\text { Yanhua Zhu }
\end{gathered}
$$

Key Laboratory of micro-inertial instrument and advanced navigation technology

Ministry of education, School of instrument science and engineering

Southeast University Nanjing 210096

tel.: +86 02583793911, fax: +8602583793911 e-mail:wlhseu@163.com CHINA 